\title{
Tuberculosis and diabetes mellitus in the Republic of Kiribati: a case-control study
}

\author{
K. Viney ${ }^{1,2}$, J. Cavanaugh ${ }^{3}$, T. Kienene ${ }^{4}$, D. Harley ${ }^{2}$, P. M. Kelly ${ }^{5,6}$, A. Sleigh ${ }^{2}$, J. O'Connor ${ }^{7}$ and S. Mase ${ }^{3}$ \\ 1 Public Health Division, Secretariat of the Pacific Community, Noumea, New Caledonia \\ 2 National Centre for Epidemiology and Population Health, Australian National University, Canberra, ACT, Australia \\ 3 Division of TB Elimination, United States Centers for Disease Control and Prevention, Atlanta, GA, USA \\ 4 National TB Programme, Ministry of Health and Medical Services, Tarawa, Kiribati \\ 5 Population Health Division, ACT Health Directorate, Canberra, ACT, Australia \\ 6 Australian National University Medical School, Canberra, ACT, Australia \\ 7 Public Health Consultant, Auckland, New Zealand
}

Abstract

objectives To better inform local management of TB-diabetes collaborative activities, we aimed to determine the prevalence of diabetes among persons with and without TB and to determine the association between TB and diabetes in Kiribati, a Pacific Island nation.

METHODS We compared consecutively enrolled TB cases to a group of randomly selected community controls without evidence of TB. Diabetes was diagnosed by HbA1c, and clinical and demographic data were collected. A tuberculin skin test was administered to controls. The chi-square test was used to assess significance in differences between cases and controls. We also calculated an odds ratio, with $95 \%$ confidence intervals, for the odds of diabetes among cases relative to controls. Unweighted multivariate logistic regression was performed to adjust for the effects of age and sex.

RESUlTs A total of $275 \mathrm{~TB}$ cases and 499 controls were enrolled. The diabetes prevalence in cases $(101,37 \%)$ was significantly greater than in controls $(94,19 \%$ ) (adjusted odds ratio: $2.8 ; 95 \%$ CI 2.0-4.1). Fifty-five percent (108) of all diabetic diagnoses were new; this proportion was higher among controls $(64.8 \%)$ than cases $(46.5 \%)$. Five patients with TB were screened to detect one patient with diabetes.

CONCLUSIONS There is a strong association between TB and diabetes in Kiribati and bidirectional screening should be conducted in this setting.

keywords Tuberculosis, diabetes mellitus, Pacific, case-control study

\section{Introduction}

Tuberculosis (TB) rates vary considerably between Pacific Island countries, ranging between 0 and 572 cases per 100000 population [1]. Similarly, diabetes is common in the Pacific, with between $7 \%$ and $38 \%$ of adults estimated to have diabetes [2-8]; the prevalence of diabetes in some Pacific Island countries is the highest in the world [2]. The Republic of Kiribati - a Pacific Island nation in the central Pacific - is burdened by both diseases, has an estimated TB incidence of 429 per 100000 population and an estimated prevalence of diabetes in adults aged 25-64 years of 29\% [1-3].

The association between TB and diabetes has been well documented; a recent systematic review of 13 studies demonstrated that diabetes is associated with a threefold risk of progression from latent TB infection (LTBI) to TB disease [9]. People with TB and diabetes may be more likely to die and have TB treatment failure or relapse than people with TB who do not have diabetes [10]. Some studies have also noted an association between diabetes and multidrug-resistant TB [11, 12], while others have not [10].

As the evidence about the association between $\mathrm{TB}$ and diabetes has grown in the last few years, international and regional policies now recommend screening patients with TB for diabetes [13, 14]. In Kiribati, the national TB programme (NTP) has not formally adopted this policy, but they have started to implement selective screening of patients with TB for diabetes, in recognition of the high rates of diabetes in the general population [3].

Although the topic has not been researched specifically, clinicians from the Kiribati NTP also report that a surprisingly large proportion of their patients with TB have concurrent diabetes. They requested assistance conducting research into this area to better inform policy about local TB-diabetes collaborative activities. As many of the 
published studies on TB and diabetes have been conducted outside of the Pacific or in countries with lower burdens of TB and diabetes, Pacific TB programme managers were also interested in local research conducted in settings with a high dual burden of disease. Therefore, we investigated the association between TB and diabetes in Kiribati, to inform local and regional policy and practice on the integration of TB and diabetes care and management.

\section{Methods}

\section{Study design}

We used an unmatched case-control study design to assess the relative odds of diabetes in residents of South Tarawa, Kiribati, with TB to those without. Our study adhered to STROBE guidelines for reporting the results of case-control studies [15].

\section{Setting}

The Republic of Kiribati is a Pacific Island country consisting of 32 coral atolls and one raised coral island (Figure 1) [16]. This study was conducted in South
Tarawa, the island on which the capital is located, and the location of the national referral hospital (Tungaru Central Hospital), the National TB Control Centre (TBCC) and the National TB Laboratory.

\section{Participants}

We estimated that to obtain a $95 \%$ confidence level with a power of $80 \%$, we would need a sample size of at least 187 cases (TB patients) and 187 controls (members of the community without symptoms of TB). Eligible TB cases were enrolled consecutively upon diagnosis between June 2010 and March 2012. The following inclusion criteria were used for cases: newly diagnosed patients with TB over 18 years old, registered for treatment at the TBCC, a resident of South Tarawa, not currently pregnant, and able to provide written informed consent. Cases were all diagnosed by experienced TB physicians using bacteriological, clinical and radiological criteria, consistent with internationally accepted definitions recommended by WHO [17].

We attempted to enrol two cases for every control. We identified our control group by first selecting enumeration areas (EAs) of South Tarawa using probability proportional to size sampling, where size was the number of

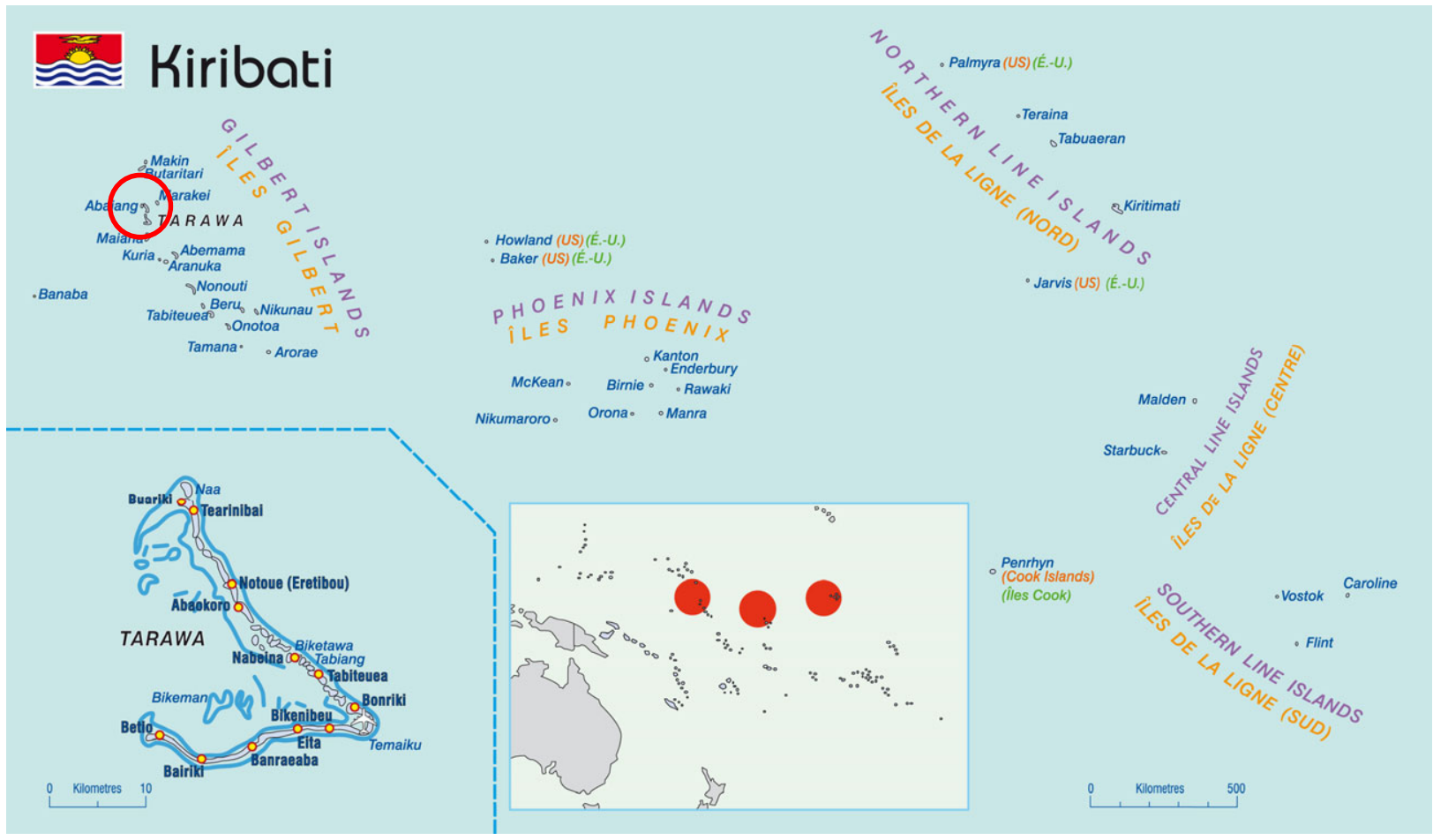

Figure I Map of the Republic of Kiribati showing its location in the Pacific Ocean (middle inset), and the study site; Tarawa (circled in red and in left inset). Source: Secretariat of the Pacific Community. 
households in the EA. We then identified 20 households in each EA using a systematic skip process. Individual controls over 18 years old were selected from identified households based on a randomly distributed list of age group and sex combinations, which was representative of the general community, as described in the 2005 census [18]. Trained nursing staff visited the selected households to introduce the study and invite participation from one control per household.

Controls were screened for TB disease by symptom review [cough (duration $>2$ weeks), fever, night sweats and/or weight loss]. Any person with one or more of these symptoms was deemed ineligible for the study and was referred for further TB evaluation.

Controls were unmatched to cases and were enrolled between August 2010 and December 2010. Other than disease status, inclusion criteria for cases and controls were identical.

\section{Data collection}

Trained nursing staff conducted the interviews of cases and controls. Structured questionnaires were used to record clinical and demographic information, including a glycosylated haemoglobin (HbA1c) test result. Patients with TB were enrolled as soon as practicable after their diagnosis and were interviewed in the $\mathrm{TB}$ ward, the $\mathrm{TB}$ clinic or at home. Controls were interviewed, had a tuberculin skin test (TST) placed and had anthropometric measurements taken at home.

\section{Measurements and definitions}

Height was measured in centimetres using a tape measure hung on a straight vertical surface. Weight, without shoes, was measured in kilograms using lightweight digital scales. Waist circumference in centimetres was determined using the tape measure wrapped around the waist at the level of the iliac crests latero-posteriorly and immediately below the umbilicus anteriorly.

The HbA1c was measured using a portable point-ofcare HbA1c test (Bayer A1c Now ${ }^{\circledR}$ : Metrika; Bayer Healthcare LLC, Sunnyvale, CA, USA) [19]. This test combines immunoassay and general chemistry [20] and was chosen due to its portable nature, ease of operation, acceptability to the population, minimally invasive method and reported clinical accuracy [21]. For the purposes of this study, any person with an $\mathrm{HbA} 1 \mathrm{c} \geq 6.5 \%$ or any person who reported a diagnosis of diabetes and treatment by a clinician was considered to have diabetes. Any person with an $\mathrm{HbA} 1 \mathrm{c} \geq 6.5 \%$ was given brief information about diabetes and was referred to the diabetes clinic at Tungaru Central Hospital, for evaluation and appropriate treatment.

After screening for active TB disease, a TST was used to assess for LTBI among controls. Five tuberculin units $(0.1 \mathrm{ml})$ of purified protein derivative were injected intradermally, and results were interpreted $48-72$ h later by measuring induration at the site of injection. An induration of $5 \mathrm{~mm}$ or more was considered positive for persons with any immunosuppressive condition (such as HIV or on immune suppressing medication), or persons with recent exposure to someone with TB; otherwise an induration of $10 \mathrm{~mm}$ or greater was considered positive. We did not perform a TST on cases, or on controls who reported a previous diagnosis of $\mathrm{TB}$, or a history of a previous positive TST.

\section{Statistical analysis}

Data were double-entered into EpiData version 3.1 and were verified and corrected prior to analysis. All analyses were carried out in EpiData Analysis version 2.2.2.178 (EpiData Association, Denmark). We calculated frequencies for categorical variables and compared them using the chi-square test; we calculated means and medians for continuous variables and compared them using the Student's $t$-test or the Wilcoxon test and Mann-Whitney U-test for nonparametric data. $P$ values $<0.05$ were considered significant. We calculated an odds ratio (OR), with $95 \%$ confidence intervals (CIs), for the odds of diabetes among cases relative to controls. Unweighted multivariate logistic regression, adjusting for age and gender, was also performed. Additionally, we estimated the proportion of TB in South Tarawa attributable to diabetes as $\left(P_{\mathrm{e}}(\mathrm{OR}-1) /\right.$ $\left.P_{\mathrm{e}}(\mathrm{OR}-1)+1\right) * 100$, where $P_{\mathrm{e}}$ was the prevalence of diabetes in the control group [22].

\section{Ethical considerations}

Ethical permission was obtained from the Institutional Review Board at the United States Centers for Disease Control and Prevention and the Human Research Ethics Committee at the Australian National University, Australia. The Government of Kiribati does not have a human research ethics committee, but the Ministry of Health and Medical Services provided approval for the study. Written and oral information was given to participants who provided written informed consent.

\section{Results}

A total of 499 controls (approximately $1 \%$ of the population of the island) were recruited from the South 
Tarawa community over the period August to December, 2010. Further, 275 TB cases were recruited from June, 2010 to March, 2012. No cases refused to participate in the study, and the refusal rate in controls was $<1 \%$. The clinical and demographic characteristics of cases and controls are outlined in Table 1.

One hundred and one (37\%) cases and 94 (19\%) controls were diabetic $(P \leq 0.001)$ (Table 2). Generally, the proportion of study participants with diabetes increased with advancing age, except in those $>65$ years. The proportion of people with diabetes in all age groups was consistently higher in cases than in controls, and the difference was statistically significant in three of the age groups (35-44, 45-54, and $\geq 65$ years). Overall, the odds of diabetes were 2.8 times higher among TB cases than controls (adjusted OR 2.8; 95\% CI 2.0-4.1; adjusted for age and sex). Cases were more likely than controls to have an $\mathrm{HbA} 1 \mathrm{c} \geq 10 \%$ (64 vs. $36 \%, P<0.001$ ).

Among the 499 controls, 220 (44\%) were current smokers and $200(40 \%)$ were obese (Body Mass Index $\left.\geq 30 \mathrm{~kg} / \mathrm{m}^{2}\right)$; among the 352 with TST data, 134

Table I Characteristics of 275 patients with TB and 499 community-based controls in Kiribati

\begin{tabular}{|c|c|c|c|}
\hline Characteristic & $\begin{array}{l}\text { Cases } \\
(n=275)\end{array}$ & $\begin{array}{l}\text { Controls } \\
(n=499)\end{array}$ & $P$-value \\
\hline $\begin{array}{l}\text { Age in years } \\
\text { mean (median) }\end{array}$ & $37.5(36)$ & $37.7(35)$ & $0.54 *$ \\
\hline $\begin{array}{l}\text { Female sex } \\
(\text { no. }-\%)\end{array}$ & $131(48)$ & $264(53)$ & 0.160 \\
\hline Mean \% HbA1c & 6.9 & 6.0 & $<0.001$ \\
\hline $\begin{array}{l}\text { HbA1c } \geq 10 \% \\
(\text { no. }-\%)\end{array}$ & $45(64)$ & $25(36)$ & $<0.001$ \\
\hline $\begin{array}{l}\text { Mean weight at } \\
\text { diagnosis } \\
\text { (kilograms) }\end{array}$ & 64.9 & 79.2 & 0.001 \\
\hline $\begin{array}{l}\text { Mean height } \\
\text { (centimetres) }\end{array}$ & 166.2 & 164.1 & 0.006 \\
\hline $\begin{array}{l}\text { Mean waist } \\
\text { circumference } \\
\text { (centimetres) }\end{array}$ & 85.5 & 96.9 & $<0.001$ \\
\hline $\begin{array}{l}\text { Mean body mass } \\
\text { index }\left(\mathrm{kg} / \mathrm{m}^{2}\right)\end{array}$ & 22.3 & 29.5 & $<0.001$ \\
\hline $\begin{array}{l}\text { Self-reported } \\
\text { comorbidities } \dagger \\
(\text { no. }-\%)\end{array}$ & $14(5)$ & $73(15)$ & $<0.001$ \\
\hline
\end{tabular}

*Wilcoxon test and Mann-Whitney U-test for nonparametric data. $\dagger$ These comorbidities included heart disease, asthma, hypertension, epilepsy, hepatitis, influenza, musculoskeletal pain, ulcers, cancer, leprosy, arthritis and dyspepsia. The number represents the number of patients who reported at least one comorbidity, and not the number of comorbidities reported; participants were able to report more than one comorbidity.
(38\%) had evidence of LTBI, as evidenced by a positive TST (Table 3). Of these, 62 (46\%) were smokers and 28 (21\%) had diabetes. Among the 64 diabetic controls with TST data, 28 (44\%) had evidence of LTBI; by comparison, 106 (37\%) of 288 non-diabetic controls with TST data had evidence of LTBI (OR 1.3; 95\% CI 0.8-2.3).

A total of 87 persons from both the case and the control groups ( $11 \%$ of the study population) had previously been diagnosed with diabetes $(20 \%$ of the TB cases $v s$. $7 \%$ of the controls, $P \leq 0.001$ ) Table 4 . Another 108 people were diagnosed with diabetes as defined for this study: $47(17 \%)$ of the cases and $61(12 \%)$ of the control group $(P<0.01)$ Table 4 . Fifty-five percent of persons with diabetes were newly diagnosed as a result of the study; five patients with TB and eight controls were screened to detect one additional person with diabetes in each group. The proportion of TB attributable to diabetes was estimated to be $25 \%$.

\section{Discussion}

This study is the first to document the association between TB and diabetes in Kiribati. We found a high prevalence of diabetes in our study population and a significant association between diabetes and TB disease. The data collected for controls provided the first information on the prevalence of LTBI in the general community of South Tarawa and provided updated information on the prevalence of diabetes, obesity and smoking, which has not been assessed since the last STEPwise approach to surveillance (STEPS) survey in 2004-2006.

In this study, the prevalence of diabetes in the community was lower than both the $28 \%$ prevalence cited in the STEPS survey and a more recent estimate of $25 \%$ among people aged 20-79 years [3, 23]; in the former, however, diabetes was diagnosed by fasting plasma glucose [3].

Consistent with studies elsewhere, patients with TB in Kiribati have nearly three times the odds of having diabetes $[9,24,25]$. In our study, $37 \%$ of patients with TB were identified as having diabetes. Prevalence estimates of diabetes in patients with TB are dependent on many factors, including the background prevalence of diabetes, genetic predisposition and the screening and diagnostic tests used to diagnose diabetes [26, 27]. Similar studies have reported a range in the proportion of patients with TB with diabetes from 9 to $44 \%$ [28-33]. In our study, $55 \%$ of the persons identified with diabetes were previously undiagnosed. This large proportion of patients newly identified with diabetes suggests that screening for diabetes in the TB clinic is a worthwhile public health intervention, provided that patients with diabetes can access diabetes care. Further, the prevalence of 
K. Viney et al. TB and diabetes in Kiribati

Table 2 Prevalence of diabetes mellitus in 275 patients with TB and 499 community-based controls, by age group in Kiribati

\begin{tabular}{lcclr}
\hline Age group (years) & $\begin{array}{c}\text { Cases }(n=275) \\
\mathrm{n}(\%)\end{array}$ & $\begin{array}{c}\text { Controls }(n=499) \\
\mathrm{n}(\%)\end{array}$ & $\begin{array}{l}\text { Odds ratio }(95 \% \\
\text { Confidence Interval) }\end{array}$ & $\begin{array}{l}P \text {-value } \\
18-24\end{array}$ \\
\hline $25-34$ & $6 / 86(7 \%)$ & $7 / 128(6 \%)$ & $1.3(0.4-4.0)$ & 0.651 \\
$35-44$ & $9 / 44(20 \%)$ & $14 / 115(12 \%)$ & $3.0(1.4-6.2)$ & 0.184 \\
$45-54$ & $21 / 45(47 \%)$ & $24 / 105(23 \%)$ & $5.1(2.4-10.9)$ & 0.004 \\
$55-64$ & $37 / 54(69 \%)$ & $23 / 77(30 \%)$ & $2.3(0.9-6.1)$ & 0.001 \\
65 and over & $20 / 30(67 \%)$ & $20 / 43(47 \%)$ & $4.2(1.1-15.7)$ & 0.030 \\
Total $^{*}$ & $8 / 16(50 \%)$ & $6 / 31(20 \%)$ & $2.5(1.8-3.5)$ & $<0.001$ \\
\hline
\end{tabular}

*Age and sex-adjusted odds ratio: 2.8 (95\% confidence interval 2.0-4.1).

undiagnosed diabetes in the controls (representative of the general population) was higher than in the cases; this may suggest that screening for diabetes in any healthcare setting may have benefits and will detect people with previously undiagnosed diabetes. In this study, we advocate screening patients with TB for diabetes as the patient is engaged with the health system for 6 months or longer (due to the duration of TB treatment) and this prolonged contact with the health system may provide a good opportunity for diagnosis and initial management of diabetes. In addition, the number of patients needed to screen to detect one case of diabetes was five and was lower than that of the general population, at eight.

The Collaborative Framework for the Care and Control of Tuberculosis and Diabetes recommends that 'patients with TB should be screened for diabetes at the start of TB treatment, where resources for diagnosis are available' [13]. The Pacific Islands Standards for the Management of Tuberculosis and Diabetes outlines six standards for managing patients with TB and diabetes. Standard 1 states that 'every person with TB over the age of 18 should be screened for diabetes mellitus' [14]. This study, one of the few in the Pacific Islands to investigate the association between TB and diabetes, supports those recommendations.

Although we cannot say from this study if diabetes preceded TB, prospective evidence supports a causal relationship between diabetes and TB [34], and other studies have rested on that assumption $[35,36]$. Assuming that diabetes preceded and was causally related to $\mathrm{TB}$, we estimate that $25 \%$ of TB in South Tarawa is attributable to diabetes. Early identification and treatment of active $\mathrm{TB}$ is a cornerstone of $\mathrm{TB}$ control and allows for improved outcomes and decreased transmission of TB; as such, patients with diabetes represent an important target group for TB screening in Kiribati. Although our study was a case-control study that does not provide direct evidence of a causal link between TB and diabetes, the association noted here and evidence from other cohort and observational studies support the adoption of recent recommendations to this effect [37-44].

Preventing the progression from LTBI to active disease is another critical component of TB control [45]. In this study, the prevalence of LTBI among controls was elevated, at $38 \%$. This is especially concerning given the high prevalence of smoking and diabetes in this group, both of which are potentially modifiable risk factors for progression from LTBI to active disease [46].

Approximately half of diabetic controls in this study had LTBI; therefore, only two diabetic patients would need to be screened to identify one case of LTBI. Screening diabetic patients for LTBI would identify a group for whom isoniazid preventive therapy could be a strategic intervention for TB control. However, this is not yet recommended and further research is required in this area.

\section{Limitations}

There are important limitations to this study. We did not have access to TB culture to verify all diagnoses of TB. Importantly, we were not able to fully assess the temporal relationship between TB and diabetes in patients with TB and were, therefore, not able to determine the causal relationship between diabetes and TB. Moreover, we did not test all patients with TB for diabetes at the same time after their TB diagnosis. HbA1c measurements vary throughout TB treatment and may be altered at the time of TB diagnosis due to inflammation, anorexia or the weight loss associated with TB, or during TB treatment by the effect of medications including oral steroids and rifampicin [47]. Conclusions about causality implicit in the population attributable fraction are based on other evidence [34], and further research is needed to determine the optimal timing for diabetes screening in patients with TB.

We assessed diabetes diagnosis using HbA1c from a portable device. We did not validate diagnosis in all individuals using another screening or diagnostic test. Although HbA1c is now recommended for the diagnosis 


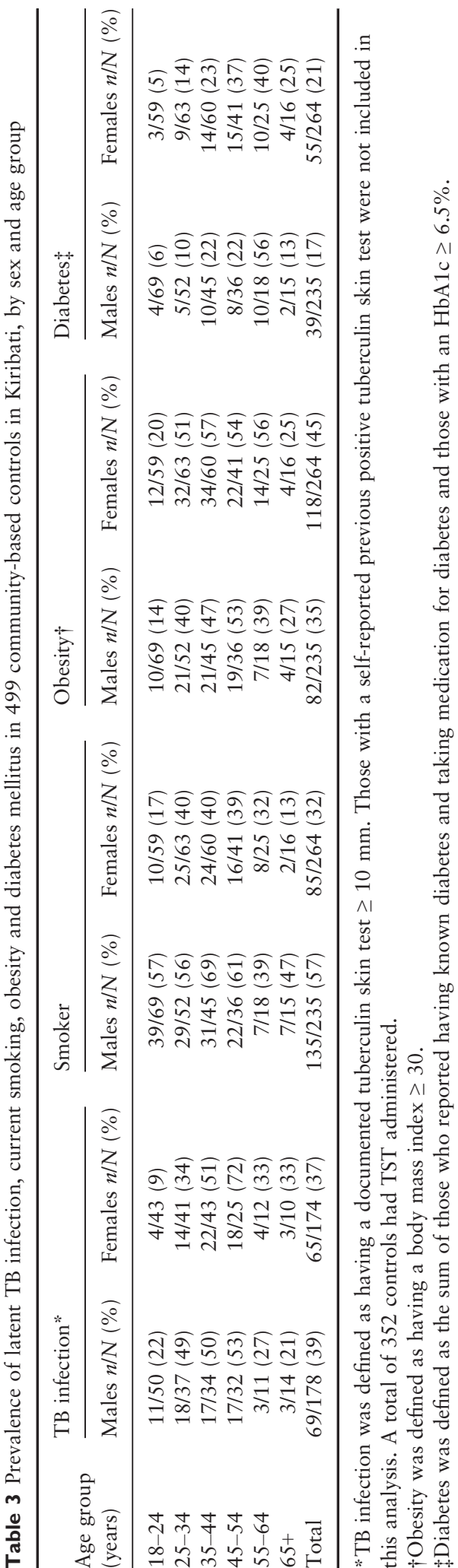

Table 4 Prevalence of previously known and newly identified diabetes mellitus in 275 patients with TB and 499 communitybased controls, in Kiribati

\begin{tabular}{lclr}
\hline Diabetes & $\begin{array}{l}\text { Cases } \\
(n=275)\end{array}$ & $\begin{array}{l}\text { Controls } \\
(n=499)\end{array}$ & $P$-value \\
\hline $\begin{array}{l}\text { Previously known } \\
\text { diabetes }\end{array}$ & $54(19.6 \%)$ & $33(7.0 \%)$ & $<0.001$ \\
$\begin{array}{l}\text { Newly identified } \\
\text { diabetes }\end{array}$ & $47(17.1 \%)$ & $61(12.0 \%)$ & 0.006 \\
$\begin{array}{l}\text { Total with } \\
\text { diabetes }\end{array}$ & $101(36.7 \%)$ & $94(18.8 \%)$ & $<0.001$ \\
$\begin{array}{l}\text { Proportion of total } \\
\text { diabetes newly } \\
\text { identified through } \\
\text { this study }\end{array}$ & $46.5 \%$ & $64.8 \%$ & - \\
\hline
\end{tabular}

of diabetes in several settings, point-of-care tests are not [48]. We compared agreement between the point-of-care HbA1c test and HbA1c testing at a laboratory in a neighbouring country for 15 persons not involved in the study, and the mean difference between the two measurements was $1 \%$. The Pearson correlation coefficient was 0.76 . It is possible that we over- or underestimated the proportion who are diabetic. The prevalence of diabetes that we report among our control patients, however, is similar to previous surveys, and our odds ratio was consistent with other studies of TB and diabetes [9]. In addition, in resource poor settings such as Kiribati, point-of-care tests for diabetes represent an important, practical and probably cost-efficient method for testing patients at risk for diabetes.

Other factors may influence HbA1c, such as chronic anaemia and haemoglobinopathies [49]. We were not able to check all participants for these conditions, and surveillance for haemoglobinopathies is not well documented in Kiribati.

Lastly, control patients in this analysis were selected using a cluster sampling scheme; we did not retain sampling variables and were not able to weight the analysis. It is possible that the precision of our CI around the odds ratio is inappropriately narrow.

\section{Conclusions}

Diabetes and TB are strongly associated with Kiribati. The high prevalence of LTBI, smoking and diabetes presents a threat to, but also an opportunity for, TB control efforts. Screening for diabetes in the TB clinic will likely increase the number of persons diagnosed with diabetes, and early diagnosis of diabetes should facilitate better management of both conditions. In settings such as Kiribati, with high TB case notification rates and a high 
prevalence of diabetes, screening persons with diabetes for TB is also justified. The Ministry of Health and Medical Services should recommend that all patients with TB be screened for diabetes and that the patients with diabetes, at a minimum, be educated about the risks and signs and symptoms of TB. We argue that collaboration between national TB and diabetes programmes should be strengthened, in much the same way that TB and HIV collaborative activities have been implemented across a range of settings.

\section{Acknowledgements}

We gratefully acknowledge the patients with TB and people from the community who willingly participated in the study, the study interviewers who interviewed the patients with TB and community members, Dr Teatao Tiira and Dr Kenneth Tabutoa (Kiribati Ministry of Health and Medical Services; Dr Tabutoa is recently deceased), and Dr Viliami Puloka (Healthy Pacific Lifestyle Team, Secretariat of the Pacific Community) who supported the implementation of the project, and the Global Fund to Fight AIDS, TB and Malaria and the Australian Aid Programme, Department of Foreign Affairs and Trade for the financial support provided the study through a dedicated operational research grant. We would like to dedicate this study to Dr Kenneth Tabutoa (now deceased and former Deputy Director of Public Health, Kiribati Ministry of Health and Medical Services) for his tireless work to manage and control TB in Kiribati. The findings and conclusions in this report are those of the authors and do not necessarily represent the official position of the Centers for Disease Control and Prevention.

\section{References}

1. World Health Organization. Global Tuberculosis Report 2013. Geneva: World Health Organization, 2013.

2. International Diabetes Federation. International Diabetes Atlas (6th edn), 2013. (Available from: http://www.idf.org/ diabetesatlas/download-book) (accessed 23 June 2014).

3. Kiribati Ministry of Health and Medical Services, World Health Organization Western Pacific Region. Kiribati NCD Risk Factors STEPS Report Tarawa, Kiribati: Kiribati Ministry of Health and Medical Services, World Health Organization, 2009.

4. Ministry of Health and Medical Services Solomon Islands, World Health Organization. Solomon Islands NCD Risk Factors STEPS Report. Suva: World Health Organization, 2010.

5. Ministry of Health Tokelau, World Health Organization. Tokelau NCD Risk Factors STEPS Report. Suva: World Health Organization, 2007.
6. Ministry of Health Fiji, World Health Organization. Fiji Non-Communicable Diseases (NCD) STEPS Survey 2002. Suva, Fiji: Ministry of Health Fiji, World Health Organization, 2004.

7. Ministry of Health Republic of Marshall Islands, World Health Organization. NCD Risk Factors STEPS Report Ministry of Health, Republic of Marshall Islands. Suva: World Health Organization, 2007.

8. Government of the Federated States of Micronesia, World Health Organization. Federated States of Micronesia (Pohnpei) NCD Risk Factors STEPS Report. Suva: World Health Organization, 2008.

9. Jeon C, Murray M. Diabetes mellitus increases the risk of active tuberculosis: a systematic review of 13 observational studies. PLoS Med 2008: 5: 1091-1101.

10. Baker M, Harries A, Jeon C et al. The impact of diabetes on tuberculosis treatment outcomes: a systematic review. BMC Med 2011: 9: 1-15.

11. Hsu A-H, Lee J-J, Chiang C-Y, Li Y-H, Chen L-K, Lin C-B. Diabetes is associated with drug resistant tuberculosis in eastern Taiwan. Int J Tuberc Lung Dis 2012: 7: 354-356.

12. Bashar M, Alcabes P, Rom W, Condos R. Increased incidence of multidrug-resistant tuberculosis in diabetic patients on the Bellevue Chest Service, 1987 to 1997. Chest 2001: 120: 1514-1519.

13. World Health Organization, International Union against Tuberculosis and Lung Disease. Collaborative Framework for the Care and Control of Tuberculosis and Diabetes Geneva. Paris: World Health Organization International Union against Tuberculosis and Lung Disease, 2011.

14. Pacific Island Tuberculosis Controller's Association. Pacific Standards for Management of TB and Diabetes. Pacific Island TB Controller's Association: Honolulu, United States of America, 2013.

15. Vandenbroucke J, von Elm E, Altman D et al. Strengthening the reporting of observational studies in epidemiology (STROBE): explanation and elaboration. PLoS Med 2007: 4: 1628-1654.

16. Kiribati Office of the President. About Kiribati, (Available from: http://www.climate.gov.ki/about-kiribati/) (accessed 2 Feb 2015).

17. World Health Organization. Treatment of Tuberculosis Guidelines (4th edn), Geneva, Switzerland: World Health Organization, 2010; 1-160.

18. Kiribati Ministry of Finance and Economic Development, Secretariat of the Pacific Community. Census 2005 Report Volume 2: Analytical Report. Noumea, New Caledonia: Kiribati Ministry of Finance and Economic Development Secretariat of the Pacific Community, 2007.

19. Bayer. BayerContour, 2013. (Available from: http:// www.bayercontour.com/Blood-Glucose-Monitoring/Classic Meters/A1CNow?WT.mc_id=\&WT.srch=1) (accessed 6 June 2013).

20. Bode B, Irvin B, Pierce J, Allen M, Clark A. Advances in hemoglobin A1c point of care technology. J Diabetes Sci Technol 2007: 1: 319-325. 
21. Chang A, Frank J, Knaebel J, Fullam J, Pardo S, Simmons D. Evaluation of an over-the-counter gylcated hemoglobin (A1C) test kit. J Diabetes Sci Technol 2010: 4: 1495-1503.

22. Webb P, Bain C, Pirozzo S. Essential Epidemiology: An Introduction for Students and Health Professionals. Cambridge: Cambridge University Press, 2005.

23. International Diabetes Federation. Western Pacific 2011. (Available from: http://www.idf.org/content/western-pacific) (accessed 07 July 2011).

24. Alisjahbana B, Van Crevel R, Sahiratmadja E et al. Diabetes mellitus is strongly associated with tuberculosis in Indonesia. Int J Tuberc Lung Dis 2006: 10: 696-700.

25. Abdelmoez B, Abd-El-Nasser A, Baheeg M, Sedky A. Prevalence of tuberculosis among children who had type 1 diabetes and were admitted to Elminia University Hospital. Pediatrics 2008: 121(Suppl): S151.

26. Marais B, Lonnroth K, Lawn S et al. Tuberculosis comorbidity with communicable and non-communicable diseases: integrating health services and control efforts. Lancet Infect Dis 2013; 13: 436-448.

27. Chen R, Corona E, Sikora M et al. Type 2 diabetes risk alleles demonstrate extreme directional differentiation among human populations, compared to other diseases. PLoS Genet 2012: 8: e1002621. doi:10.1371/journal. pgen.1002621.

28. Restrepo B, Camerlin A, Rahbar M et al. Cross-sectional assessment reveals high diabetes prevalence among newlydiagnosed tuberculosis cases. Bull World Health Organ 2011: 89: 352-359.

29. Balakrishnan S, Vijayan S, Nair S et al. High diabetes prevalence among tuberculosis cases in Kerala, India. PLoS One 2012: 7: e46502. doi:10.1371/journal.pone.0046502

30. Vishwanathan V, Kumpatla S, Aravindalochanan V et al. Prevalence of diabetes and pre-diabetes associated risk factors among tuberculosis patients in India. PLoS One 2012: 7: e41367. doi:10.1371/journal.pone.0041367.

31. Alladin B, Mack S, Singh A et al. Tuberculosis and diabetes in Guyana. Int J Infect Dis 2011: 15: e818-e821.

32. Li L, Lin Y, Tan S et al. Screening of patients with tuberculosis for diabetes mellitus in China. Trop Med Int Health 2012: 17: 1294-1301.

33. Kibirige D, Ssekitoleko R, Mutebi E, Worodria W. Overt diabetes mellitus among newly diagnosed Ugandan tuberculosis patients: a cross sectional study. BMC Infect Dis 2013: 13: 122. doi:10.1186/1471-2334-13-122.

34. Dooley K, Chaisson R. Tuberculosis and diabetes mellitus: convergence of two epidemics. Lancet Infect Dis 2009: 9: 737-746.
35. Pablos-Mendez A, Blustein J, Knirsch C. The role of diabetes mellitus in the higher prevalence of tuberculosis among Hispanics. Am J Public Health 1997: 87: 574-579.

36. Faurholt-Jepsen D, Range N, PrayGod G et al. Diabetes is a risk factor for pulmonary tuberculosis: a case-control study from Mwanza, Tanzania. PLoS One 2011: 8: e24215. doi:10.1371/journal.pone.0024215

37. World Health Organization. Systematic Screening for Active Tuberculosis: Principles and Recommendations. Geneva: World Health Organization, 2013; i-133.

38. Lin Y, Li L, Mi F et al. Screening patients with diabetes mellitus for tuberculosis in China. Trop Med Int Health 2012: 17: 1302-1308.

39. Kermansaravi F, Metanat M, Sharifi-Mood B. Evaluation of active pulmonary tuberculosis among patients with diabetes. Int J Infect 2014: 1: e19632.

40. Amare H, Gelaw A, Anagaw B, Gelaw B. Smear positive pulmonary tuberculosis among diabetic patients at the Dessie referral hospital, Northeast Ethiopia. Infect Dis Poverty 2013: 2. doi:10.1186/2049-9957-2-6.

41. Wang Q, Ma A, Han X et al. Prevalence of type 2 diabetes among newly detected pulmonary tuberculosis patients in China: a community based cohort study. PLoS One 2013: 8: e82660.

42. Prakash B, Ravish K, Prabhakar B et al. Tuberculosis-diabetes mellitus bidirectional screening at a tertiary care centre, South India. Pub Health Act 2013: 3(Suppl 1): 18-22.

43. India Diabetes Mellitus-Tuberculosis Study Group. Screening of patients with diabetes mellitus for tuberculosis in India. Trop Med Int Health 2013; 18: 646-654.

44. Jeon C, Harries A, Baker MA et al. Bi-directional screening for tuberculosis and diabetes: a systematic review. Trop Med Int Health 2010: 15: 1300-1314.

45. Lonnroth K, Corbett E, Golub J et al. Systematic screening for active tuberculosis: rationale, definitions and key considerations. Int J Tuberc Lung Dis 2013: 17: 289-298.

46. Lönnroth K, Jaramillo E, Williams B, Dye C, Raviglione M. Drivers of tuberculosis epidemics: the role of risk factors and social determinants. Soc Sci Med 2009: 68: 2240-2246.

47. Oluboyo P, Erasmus R. The significance of glucose intolerance in pulmonary tuberculosis. Tubercle 1990: 71: 135138.

48. Committee TIE. International Expert Committee report on the role of A1c assay in the diagnosis of diabetes. Diabetes Care 2009: 32: 1327-1334.

49. Bonora E, Tuomilehto J. The pros and cons of diagnosing diabetes with A1c. Diabetes Care 2011: 34(Suppl 2): S184 S190.

Corresponding Author Kerri Viney, National Centre for Epidemiology and Population Health, Research School of Population Health, Australian National University, Building 62, Cnr Mills and Eggleston Roads, Canberra ACT 0200, Australia.

E-mail: kerri.viney@hotmail.com 